\title{
A Highly Selective and Efficient Method for the Preparation of Amides from Carboxylic Acids and Amines Using Sodium Hydrogen Sulfate Adsorbed on Silica Gel ${ }^{\dagger}$
}

\author{
Rathod Aravind Kumar ${ }^{1,3}$, Nayaki Salvanna ${ }^{1,2}$, Biswanath Das ${ }^{1, \text { * }, ~ J a j u l a ~ K a s h a n n a ~}{ }^{1,4, ~ * ~}$ \\ ${ }^{1}$ Natural Products Chemistry Division, CSIR- Indian Institute of Chemical Technology, Hyderabad, India \\ ${ }^{2}$ Osmania University Post Graduate College, Medak, India \\ ${ }^{3}$ SemiochemicalDivision, CSIR- Indian Institute of Chemical Technology, Hyderabad, India \\ ${ }^{4}$ Department of Chemistry, Rajiv Gandhi University of Knowledge Technologie, Basar, India
}

Email address:

jajulakashanna@yahoo.co.in (J. Kashanna)

${ }^{*}$ Corresponding author

\section{To cite this article:}

Rathod Aravind Kumar, Nayaki Salvanna, Biswanath Das, Jajula Kashanna. A Highly Selective and Efficient Method for the Preparation of Amides from Carboxylic Acids and Amines Using Sodium Hydrogen Sulfate Adsorbed on Silica Gel †. World Journal of Applied Chemistry. Vol. 4, No. 1, 2019, pp. 14-18. doi: 10.11648/j.wjac.20190401.13

Received: February 23, 2019; Accepted: April 23, 2019; Published: June 17, 2019

\begin{abstract}
The formation of amides from carboxylic acids and amines has been catalyzed efficiently with sodium hydrogen sulfate adsorbed on silica gel $\left(\mathrm{NaHSO}_{4} \cdot \mathrm{SiO}_{2}\right)$ at room temperature to give the products in high yields. The conversion carried out under reflux requires less reaction times and forms the products in higher yields. The reaction is highly selective as it has been found that either or both of the substrates should be aliphatic but it is failure when the acid as well as the amine is aromatic. The method has been utilized for the preparation of a natural phenethyl amide derivative and its analogues.
\end{abstract}

Keywords: Heterogeneous Catalysis, Carboxylic Acids, Amines, Amides, Nucleophilic Substitution

\section{Introduction}

Amides are useful intermediates in various reactions of carboxylic acids and amines in which it is desirable to protect these groups [1-3]. Amides can easily be hydrolyzed by acids or alkalis to generate the parent amines and carboxylic acids. Several amides are used in medicine as febrifuge [1]. The amide constituents isolated from nature have been found to possess various biological properties including anticancer activity [4-5]. The amides can also be converted into esters, amines and nitriles [1]. The former are generally prepared by the action of amines with acid chlorides, acid anhydrides or ester [1-6]. The direct conversion of esters into amides is a useful synthetic procedure but the aminolysis of esters generally requires high temperature and the catalyst used here may affect other functionalities. The one-step conversion of carboxylic acids into amides can also be effected with various reagents [7-24]. However, many of these reagents are not easily available and expensive. The preparation of these reagents also involves complex steps. Moreover, some of the reagents requires tedious experimental procedures and long reaction times and form the products with low yields. The selectivity of the amide formation by using these reagents has also been examined only in a few cases [21]. Thus there is a need to develop a simple, efficient and selective method for preparation of amides.

\section{Experimental Section}

\subsection{Materials and Method}

The spectra were recorded with the following instruments: IR: Perkin Elmer RX1 FT-IR spectrophotometer; NMR: Varian Gemini $200 \mathrm{MHz}$ and ESI-MS: LC-MSD Trap SL spectrometer, Column Chromatography was carried out over silica gel (BDH 100-200 Mesh) and TLC was performed with silica gel GF 254 (Merck) plates. 


\subsection{General Experimental Procedure for Preparation of Amides}

To a solution of an amine $(1.2 \mathrm{mmol})$ dissolved in $\mathrm{CHCl}_{3}$ or $\mathrm{CH}_{2} \mathrm{Cl}_{2}(10 \mathrm{ml})$ an acid $(1 \mathrm{mmol})$ and the catalyst, $\mathrm{NaHSO}_{4} \cdot \mathrm{SiO}_{2}(100 \mathrm{mg})$ were added. The mixture was stirred at room temperature or under reflux. The reaction was monitored by TLC. After completion the solvent was removed and water $(10 \mathrm{ml})$ was added. The mixture was extracted with EtOAc $(3 \mathrm{x}$ $10 \mathrm{ml}$ ) and the extract was dried, concentrated and subjected to column chromatography to obtain a pure amide.

\section{Results and Discussion}

In continuation of our work [26-33] on the application of silica gel supported sodium hydrogen sulfate $\left(\mathrm{NaHSO}_{4} \cdot \mathrm{SiO}_{2}\right)$ catalyst for synthesis of bioactive compounds as well as for developments of novel synthetic methodologies we have observed that the catalyst can efficiently be utilized for onestep preparation of amides from carboxylic acids and amines (Figure 1).

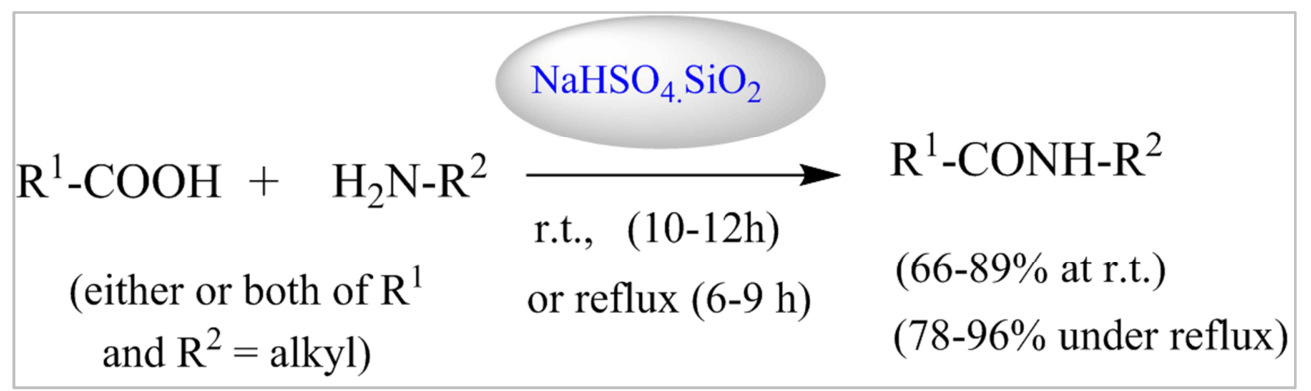

Figure 1. One pot reaction of carboxylic acids and amines in the presence of sodium hydrogen sulphate adsorbed on silica gel at room temperature orunder reflux.

A series of amides has been prepared by $\mathrm{NaHSO}_{4} \cdot \mathrm{SiO}_{2}$ catalyzed reaction of acids and amines at room temperature (Table 1). The yields of amides are high. When the conversion was carried out under reflux in $\mathrm{CHCl}_{3}$ or toluene the time required for amide formation was less and the yields of the amides were higher (Table 1).

The amides were prepared from different aromatic and aliphatic acids and amines. The aromatic acids and anilines contained both electron-donating and electron-withdrawing groups. Long chain aliphatic acids also underwent the conversion smoothly. Various functional groups such as halogen, hydroxyl, ether and nitro remained intact. The structures of the amides were established from their spectral (IR, ${ }^{1} \mathrm{H}$ and ${ }^{13} \mathrm{C} \mathrm{NMR}$ and MS) and analytical data.

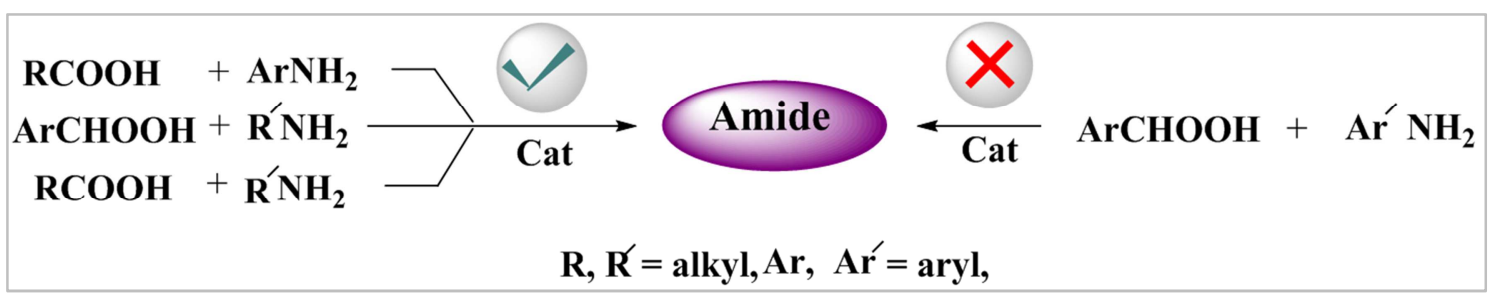

Figure 2. Selectivity in the preparation of amides using $\mathrm{NaHSO}_{4} \cdot \mathrm{SiO}_{2}$

The selectivity of the present conversion is interesting (Figure 2). It has been observed that at least one of the acid and amine should be aliphatic for the formation of an amide. However, if both are aromatic the reaction will not occur (Table 1)

Table 1. Formation of amides from carboxylic acids and amines ${ }^{a}$.

\begin{tabular}{|c|c|c|c|c|c|c|}
\hline \multirow{2}{*}{ Entry } & \multirow{2}{*}{ Acid } & \multirow{2}{*}{ Amine } & \multicolumn{2}{|l|}{ At r.t. } & \multicolumn{2}{|c|}{ Under reflux } \\
\hline & & & Time (h) & Isolated yield (\%) & Time (h) & Isolated yield (\%) \\
\hline 1 & $\mathrm{CH}_{3} \mathrm{CO}_{2} \mathrm{H}$ & $\mathrm{C}_{6} \mathrm{H}_{5} \mathrm{NH}_{2}$ & 10 & 75 & 8 & 92 \\
\hline 2 & $\mathrm{CH}_{3} \mathrm{CO}_{2} \mathrm{H}$ & 4-(F) $\mathrm{C}_{6} \mathrm{H}_{4} \mathrm{NH}_{2}$ & 11 & 80 & 9 & 93 \\
\hline 3 & $\mathrm{CH}_{3} \mathrm{CO}_{2} \mathrm{H}$ & $2,3-(\mathrm{Cl})_{2} \mathrm{C}_{6} \mathrm{H}_{3} \mathrm{NH}_{2}$ & 11 & 85 & 7 & 91 \\
\hline 4 & $\mathrm{CH}_{3} \mathrm{CO}_{2} \mathrm{H}$ & $2,4,6-(\mathrm{Cl})_{3} \mathrm{C}_{6} \mathrm{H}_{2} \mathrm{NH}_{2}$ & 11 & 79 & 9 & 82 \\
\hline 5 & $\mathrm{CH}_{3} \mathrm{CH}_{2} \mathrm{CO}_{2} \mathrm{H}$ & $3-(\mathrm{Me}) \mathrm{C}_{6} \mathrm{H}_{4} \mathrm{NH}_{2}$ & 12 & 82 & 8 & 91 \\
\hline 6 & $\mathrm{CH}_{3} \mathrm{CH}_{2} \mathrm{CO}_{2} \mathrm{H}$ & $2,3-(\mathrm{Cl})_{2} \mathrm{C}_{6} \mathrm{H}_{3} \mathrm{NH}_{2}$ & 10 & 69 & 8 & 87 \\
\hline 8 & $\mathrm{C}_{6} \mathrm{H}_{5} \mathrm{CH}_{2} \mathrm{CO}_{2} \mathrm{H}$ & $\mathrm{C}_{6} \mathrm{H}_{5} \mathrm{NH}_{2}$ & 11 & 65 & 7 & 94 \\
\hline 9 & 3- $(\mathrm{Cl}) \mathrm{C}_{6} \mathrm{H}_{4} \mathrm{CH}_{2} \mathrm{CO}_{2} \mathrm{H}$ & $2,3-(\mathrm{Cl})_{2} \mathrm{C}_{6} \mathrm{H}_{3} \mathrm{NH}_{2}$ & 12 & 69 & 8 & 96 \\
\hline 10 & $\mathrm{CH}_{3}\left(\mathrm{CH}_{2}\right)_{8} \mathrm{CO}_{2} \mathrm{H}$ & $2,3-(\mathrm{Cl})_{2} \mathrm{C}_{6} \mathrm{H}_{3} \mathrm{NH}_{2}$ & 12 & 69 & 9 & 80 \\
\hline 11 & $\mathrm{C}_{6} \mathrm{H}_{5} \mathrm{CO}_{2} \mathrm{H}$ & $\mathrm{CH}_{3} \mathrm{NH}_{2}$ & 11 & 76 & 6 & 92 \\
\hline 12 & $4-(\mathrm{OH}) \mathrm{C}_{6} \mathrm{H}_{4} \mathrm{CO}_{2} \mathrm{H}$ & $\mathrm{CH}_{3} \mathrm{NH}_{2}$ & 12 & 79 & 7 & 84 \\
\hline 13 & $2-(\mathrm{Cl}) \mathrm{C}_{6} \mathrm{H}_{4} \mathrm{CO}_{2} \mathrm{H}$ & $\mathrm{CH}_{3} \mathrm{NH}_{2}$ & 11 & 78 & 8 & 83 \\
\hline 14 & $2-(\mathrm{I}) \mathrm{C}_{6} \mathrm{H}_{4} \mathrm{CO}_{2} \mathrm{H}$ & $\mathrm{CH}_{3} \mathrm{NH}_{2}$ & 10 & 69 & 8 & 78 \\
\hline
\end{tabular}




\begin{tabular}{|c|c|c|c|c|c|c|}
\hline \multirow{2}{*}{ Entry } & \multirow{2}{*}{ Acid } & \multirow{2}{*}{ Amine } & \multicolumn{2}{|l|}{ At r.t. } & \multicolumn{2}{|c|}{ Under reflux } \\
\hline & & & Time (h) & Isolated yield (\%) & Time (h) & Isolated yield (\%) \\
\hline 15 & 4- $\left(\mathrm{NO}_{2}\right) \mathrm{C}_{6} \mathrm{H}_{4} \mathrm{CO}_{2} \mathrm{H}$ & $\mathrm{CH}_{3} \mathrm{NH}_{2}$ & 12 & 66 & 7 & 79 \\
\hline 16 & $3,5-(\mathrm{OH})_{2} \mathrm{C}_{6} \mathrm{H}_{4} \mathrm{CO}_{2} \mathrm{H}$ & $\mathrm{CH}_{3} \mathrm{NH}_{2}$ & 12 & 78 & 9 & 84 \\
\hline 17 & $3,5-(\mathrm{Br})_{2} \mathrm{C}_{6} \mathrm{H}_{4} \mathrm{CO}_{2} \mathrm{H}$ & $\mathrm{CH}_{3} \mathrm{NH}_{2}$ & 12 & 80 & 9 & 89 \\
\hline 18 & $3,5-\left(\mathrm{NO}_{2}\right)_{2} \mathrm{C}_{6} \mathrm{H}_{4} \mathrm{CO}_{2} \mathrm{H}$ & $\mathrm{C}_{6} \mathrm{H}_{5} \mathrm{CH}_{2} \mathrm{NH}_{2}$ & 11 & 75 & 9 & 81 \\
\hline 19 & $\mathrm{CH}_{3} \mathrm{CH}_{2} \mathrm{CO}_{2} \mathrm{H}$ & $\mathrm{CH}_{3} \mathrm{NH}_{2}$ & 11 & 66 & 8 & 82 \\
\hline 20 & $\mathrm{CH}_{3} \mathrm{CH}_{2} \mathrm{CO}_{2} \mathrm{H}$ & $\mathrm{CH}_{3}\left(\mathrm{CH}_{2}\right)_{2} \mathrm{NH}_{2}$ & 11 & 77 & 9 & 81 \\
\hline 21 & $\mathrm{CH}_{3}\left(\mathrm{CH}_{2}\right)_{8} \mathrm{CO}_{2} \mathrm{H}$ & $\mathrm{CH}_{3} \mathrm{NH}_{2}$ & 10 & 72 & 8 & 89 \\
\hline 22 & $\mathrm{CH}_{3} \mathrm{CO}_{2} \mathrm{H}$ & $\mathrm{C}_{6} \mathrm{H}_{5} \mathrm{CH}_{2} \mathrm{NH}_{2}$ & 11 & 77 & 8 & 82 \\
\hline 23 & $\mathrm{CH}_{3} \mathrm{CH}_{2} \mathrm{CO}_{2} \mathrm{H}$ & 4-(OH) $\mathrm{C}_{6} \mathrm{H}_{5}\left(\mathrm{CH}_{2}\right)_{2} \mathrm{NH}_{2}$ & 12 & 66 & 9 & 78 \\
\hline 24 & $\mathrm{CH}_{3} \mathrm{CH}_{2} \mathrm{CO}_{2} \mathrm{H}$ & $\mathrm{C}_{6} \mathrm{H}_{5}\left(\mathrm{CH}_{2}\right)_{2} \mathrm{NH}_{2}$ & 11 & 80 & 9 & 90 \\
\hline 25 & $\mathrm{CH}_{3}\left(\mathrm{CH}_{2}\right)_{8} \mathrm{CO}_{2} \mathrm{H}$ & $\mathrm{C}_{6} \mathrm{H}_{5} \mathrm{CH}_{2} \mathrm{NH}_{2}$ & 11 & 85 & 8 & 93 \\
\hline 26 & $\mathrm{CH}_{3} \mathrm{CH}(\mathrm{Cl}) \mathrm{CO}_{2} \mathrm{H}$ & $\mathrm{C}_{6} \mathrm{H}_{5} \mathrm{CH}_{2} \mathrm{NH}_{2}$ & 11 & 82 & 8 & 88 \\
\hline 27 & $\mathrm{C}_{6} \mathrm{H}_{6} \mathrm{CO}_{2} \mathrm{H}$ & $\mathrm{C}_{6} \mathrm{H}_{5} \mathrm{NH}_{2}$ & 12 & 0 & 9 & 0 \\
\hline 28 & $\mathrm{C}_{6} \mathrm{H}_{6} \mathrm{CO}_{2} \mathrm{H}$ & 2-( $\left(\mathrm{NO}_{2}\right) \mathrm{C}_{6} \mathrm{H}_{4} \mathrm{NH}_{2}$ & 12 & 0 & 9 & 0 \\
\hline 29 & 3-(Cl) $\mathrm{C}_{6} \mathrm{H}_{4} \mathrm{CO}_{2} \mathrm{H}$ & 4-(OH) $\mathrm{C}_{6} \mathrm{H}_{4} \mathrm{NH}_{2}$ & 12 & 0 & 9 & 0 \\
\hline 30 & $2-(\mathrm{I}) \mathrm{C}_{6} \mathrm{H}_{4} \mathrm{CO}_{2} \mathrm{H}$ & $4-(\mathrm{Me}) \mathrm{C}_{6} \mathrm{H}_{4} \mathrm{NH}_{2}$ & 12 & 0 & 9 & 0 \\
\hline 31 & $2-\left(\mathrm{NH}_{2}\right) \mathrm{C}_{6} \mathrm{H}_{4} \mathrm{CO}_{2} \mathrm{H}$ & $\mathrm{C}_{6} \mathrm{H}_{5} \mathrm{NH}_{2}$ & 12 & 0 & 9 & 0 \\
\hline 32 & 4- $\left(\mathrm{NO}_{2}\right) \mathrm{C}_{6} \mathrm{H}_{4} \mathrm{CO}_{2} \mathrm{H}$ & $\mathrm{C}_{6} \mathrm{H}_{5} \mathrm{NH}_{2}$ & 12 & 0 & 9 & 0 \\
\hline 33 & $3,5-\left(\mathrm{NO}_{2}\right) \mathrm{C}_{6} \mathrm{H}_{3} \mathrm{CO}_{2} \mathrm{H}$ & 4-(OMe) $\mathrm{C}_{6} \mathrm{H}_{4} \mathrm{NH}_{2}$ & 12 & 0 & 9 & 0 \\
\hline
\end{tabular}

${ }^{\mathrm{a}}$ The structures of the products were established from the spectral (IR, ${ }^{1} \mathrm{H}$ and ${ }^{13} \mathrm{C}$ NMR and MS) and analytical data.

The present method has been applied for the synthesis of natural tyramide, $\mathrm{N}$ - (4- hydroxyphenethyle) propionamide (Table 1, entry 23). The compound occurs in marine bacteria [34] and Myrmicine ants [35]. It has also been obtained as a metabolite of an endophytic Stryptomycessp [36]. The starting material, 4-hydroxyphenethyle amine was prepared from 4-hydroxyphenyl methanol by converting it into the corresponding nitrile by treatment with $\mathrm{NaCN}$ in DMF [37] followed by reduction of the product with $\mathrm{NaBH}_{4} / \mathrm{I}_{2}$ system [38]. Treatment of 4-hydroxyphenethyl amine with propionic acid in the presence of $\mathrm{NaHSO}_{4} \cdot \mathrm{SiO}_{2}$ at room temperature or under reflux afforded $\mathrm{N}$ - (4-hydroxphenethyl) propionamide. Some analogues of this compound (entry 20, 22, 24, 25 and 26) have also been prepared following the similar method.

The catalyst, $\mathrm{NaHSO}_{4} \cdot \mathrm{SiO}_{2}$ works under heterogeneous conditions. In recent years heterogeneous catalysts have attracted much attention due to eco-economic benefits. The catalysts can easily be prepared [39] from its readily available ingredient, $\mathrm{NaHSO}_{4}$ and silica gel (100-200 mesh). It can also easily be removed from the reaction mixture.

\section{Conclusion}

In conclusion, we have developed a novel and efficient methodology for one-pot preparation of amides from carboxylic acids and amines (both should not be aromatic) in the presence of $\mathrm{NaHSO}_{4} \cdot \mathrm{SiO}_{2}$ as a catalyst. The procedure offers several advantages including mild reaction conditions, operational simplicity, high selectivity and utilization of a non-expensive and non-hazardous heterogeneous catalyst. The method will be useful for separation of aromatic carboxylic acids from a mixture containing aliphatic carboxylic acids as well as for separation of aromatic amines from a mixture containing aliphatic amines. The method has successfully been applied for the synthesis of natural tyramide, $\mathrm{N}$ - (4- hydroxyphenethyle) propionamide and its analogues.

The spectral and analytical data of some representative amides are given below:

$N$-m-Tolylpropionamide (Table 1, Entry 5):

IR (KBr): $v=3305,1662,1607,1547,1437,1217 \mathrm{~cm}^{-1}$; ${ }^{1} \mathrm{H}$ NMR $\left(200 \mathrm{MHz}, \mathrm{CDCl}_{3}\right): \delta=7.36-7.21(3 \mathrm{H}, \mathrm{m}), 7.10$ $(1 \mathrm{H}, \mathrm{t}, J=8.0 \mathrm{~Hz}), 6.82(1 \mathrm{H}, \mathrm{d}, J=8.0 \mathrm{~Hz}), 2.40-2.21(5 \mathrm{H}$, m), $1.10(3 \mathrm{H}, \mathrm{t}, J=7.0 \mathrm{~Hz}) ;{ }^{13} \mathrm{C} \mathrm{NMR}\left(50 \mathrm{MHz}, \mathrm{CDCl}_{3}\right): \delta=$ 173.8, 138.2, 138.0, 128.4, 124.8, 121.0, 116.9, 30.1, 20.6; ESIMS: $\mathrm{m} / \mathrm{z} 164[\mathrm{M}+\mathrm{H}]^{+}, 186[\mathrm{M}+\mathrm{Na}]^{+}$; anal. Calcd for $\mathrm{C}_{10} \mathrm{H}_{13} \mathrm{NO}$ : $\mathrm{C} 73.62 ; \mathrm{H} 7.98$; $\mathrm{N}$ 8.59; found: C 73.71; H 7.92; N 8.53.

$N$-(2,3-Dichlorophenyl) propionamide (Table, Entry 6):

IR (KBr): $v=3291,1669,1579,1523,1406,1366,1296$ $\mathrm{cm}^{-1} ;{ }^{1} \mathrm{H}$ NMR $\left(200 \mathrm{MHz}, \mathrm{CDCl}_{3}\right): \delta=8.32(1 \mathrm{H}, \mathrm{t}, J=8.0$ $\mathrm{Hz}), 7.72$ (1H, brs), $7.17(2 \mathrm{H}, \mathrm{d}, J=8.0 \mathrm{~Hz}), 2.42(2 \mathrm{H}, \mathrm{t}, J=$ $7.0 \mathrm{~Hz}), 1.80-1.69(2 \mathrm{H}, \mathrm{m}) 1.41-1.20(12 \mathrm{H}, \mathrm{m}) 0.83(3 \mathrm{H}, \mathrm{t}$, $J=7.0 \mathrm{~Hz}) ;{ }^{13} \mathrm{C}$ NMR $\left(50 \mathrm{MHz}, \mathrm{CDCl}_{3}\right): \delta=171.7,136.8$, 133.0, 127.9, 125.0, 121.0, 119.9, 38.1, 32.2, 29.7, 29.0, 25.3, 22.6, 14.2; ESIMS: m/z 316, 318, $320[\mathrm{M}+\mathrm{H}]^{+}, 338,340,342$ $[\mathrm{M}+\mathrm{Na}]^{+}$; anal. Calcd for $\mathrm{C}_{16} \mathrm{H}_{23} \mathrm{Cl}_{2} \mathrm{NO}$ : $\mathrm{C} 60.76 ; \mathrm{H} 7.28 ; \mathrm{N}$ 4.43; found: $\mathrm{C} 60.84 ; \mathrm{H} 7.32 ; \mathrm{N} 4.47$.

2-(2-Chlorophenyl)- $N$-(2,3-dichlorophenyl) acetamide (Table 1, Entry 9):

IR (KBr): $v=3293,1645,1550,1453,1368,1281 \mathrm{~cm}^{-1} ;{ }^{1} \mathrm{H}$ NMR $\left(200 \mathrm{MHz}, \mathrm{CDCl}_{3}\right): \delta=8.32(1 \mathrm{H}, \mathrm{dd}, J=8.0,2.0 \mathrm{~Hz})$, 7.80 (1H, brs), $7.51-7.40$ (3H, m), $7.38-7.30$ (2H, m), 7.19 $(2 \mathrm{H}, \mathrm{d}, J=8.0 \mathrm{~Hz}), 3.92(1 \mathrm{H}, \mathrm{s}) ;{ }^{13} \mathrm{C} \mathrm{NMR}(50 \mathrm{MHz}$, $\left.\mathrm{CDCl}_{3}\right): \delta=168.0,136.7,135.0,132.1,132.0,130.2,130.0$, 127.9, 125.4, 119.5, 42.7, ESIMS: m/z 314, 316, 318, 320 $[\mathrm{M}+\mathrm{H}]^{+}, \quad 336, \quad 340,342[\mathrm{M}+\mathrm{Na}]^{+} ;$anal. Calcd for $\mathrm{C}_{14} \mathrm{H}_{10} \mathrm{Cl}_{3} \mathrm{NO}$ : C 53.42; $\mathrm{H}$ 3.18; $\mathrm{N} 4.45$; found: $\mathrm{C} 53.51 ; \mathrm{H}$ $3.22 ; \mathrm{N} 4.51$.

2-Iodo- $N$-Methylbenzamide (Table 1, Entry 14):

IR (KBr): $v=3436,1654,1565,1510,1440 \mathrm{~cm}^{-1} ;{ }^{1} \mathrm{H}$ NMR $\left(200 \mathrm{MHz}, \mathrm{CDCl}_{3}\right): \delta=7.98(1 \mathrm{H}, \mathrm{d}, J=8.0 \mathrm{~Hz}) 7.58$ $(1 \mathrm{H}, \mathrm{brs}), 7.40(1 \mathrm{H}, \mathrm{t}, J=8.0 \mathrm{~Hz}), 6.67(1 \mathrm{H}, \mathrm{d}, J=8.0 \mathrm{~Hz})$, $6.59(1 \mathrm{H}, \mathrm{t}, J=8.0 \mathrm{~Hz}), 2.94(3 \mathrm{H}, \mathrm{s}) ;{ }^{13} \mathrm{C} \mathrm{NMR}(50 \mathrm{MHz}$, 
$\left.\mathrm{CDCl}_{3}\right): \delta=167.9,131.8,130.1,129.5,127.8,125.3,119.2$, 42.9; ESIMS: $\mathrm{m} / \mathrm{z} 128[\mathrm{M}+\mathrm{H}]^{+}$; anal. Calcd for $\mathrm{C}_{8} \mathrm{H}_{8} \mathrm{INO}: \mathrm{C}$ 36.78; H 3.07; N 5.36; found: C 36.86; H 3.12; N 5.27.

2,6-Dibromo- $N$-methylbenzamide (Table 1, Entry 17):

IR $(\mathrm{KBr}): v=3425,1662,1590,1450,1416,1364 \mathrm{~cm}^{-1}$; ${ }^{1} \mathrm{H}$ NMR $\left(200 \mathrm{MHz}, \mathrm{CDCl}_{3}\right): \delta=8.02(2 \mathrm{H}, \mathrm{s}), 7.63(1 \mathrm{H}, \mathrm{s})$, $6.12(1 \mathrm{H}$, brs $), 2.49(3 \mathrm{H}, \mathrm{S}) ;{ }^{13} \mathrm{C} \mathrm{NMR}\left(50 \mathrm{MHz}, \mathrm{CDCl}_{3}\right): \delta=$ 167.0, 139.4, 133.9, 130.0, 121.0, 23.5; ESIMS: m/z 292, 294, $296[\mathrm{M}+\mathrm{H}]^{+}$; anal. Calcd for $\mathrm{C}_{8} \mathrm{H}_{7} \mathrm{Br}_{2} \mathrm{NO}$ : $\mathrm{C}$ 32.76; $\mathrm{H}$ 2.39; N 4.78; found: C 32.68; H 2.42; N 4.83.

$N$-Methylpropionamide (Table 1, Entry 21):

IR (KBr): $v=3300,1638,1563,1416,1375,1290 \mathrm{~cm}^{-1}$; ${ }^{1} \mathrm{H}$ NMR $\left(200 \mathrm{MHz}, \mathrm{CDCl}_{3}\right): \delta=6.12(1 \mathrm{H}, \mathrm{brs}), 2.79(3 \mathrm{H}, \mathrm{s})$, $2.12(2 \mathrm{H}, \mathrm{t}, J=7.0 \mathrm{~Hz}), 1.67-1.52(2 \mathrm{H}, \mathrm{m}), 1.38-1.20$ $(12 \mathrm{H}, \mathrm{m}), 0.88(3 \mathrm{H}, \mathrm{t}, J=7.0 \mathrm{~Hz}) ;{ }^{13} \mathrm{C} \mathrm{NMR}(50 \mathrm{MHz}$, $\left.\mathrm{CDCl}_{3}\right): \delta=173.8,36.9,32.2,29.8,29.3,26.2,26.0,20.9$, 14.1; ESIMS: m/z $186[\mathrm{M}+\mathrm{H}]^{+}, 208[\mathrm{M}+\mathrm{Na}]^{+}$; anal. Calcd for $\mathrm{C}_{11} \mathrm{H}_{23} \mathrm{NO}$ : $\mathrm{C} 71.35$; $\mathrm{H} 12.43$; $\mathrm{N} 7.57$; found: $\mathrm{C} 71.44 ; \mathrm{H}$ 12.48; N 7.49.

$N$-Benzylacetamide (Table 1, Entry 22):

IR (KBr): $v=3293,1647,1551,1454,1375,1281 \mathrm{~cm}^{-1} ;{ }^{1} \mathrm{H}$ NMR $\left(200 \mathrm{MHz}, \mathrm{CDCl}_{3}\right): \delta=7.28-7.15(5 \mathrm{H}, \mathrm{m}), 6.83(1 \mathrm{H}$, brs), $4.30(2 \mathrm{H}, \mathrm{s}), 1.92(3 \mathrm{H}, \mathrm{S}) ;{ }^{13} \mathrm{C} \mathrm{NMR}\left(50 \mathrm{MHz}, \mathrm{CDCl}_{3}\right)$ : $\delta=170.8,137.9,128.4,127.5,127.2,43.4,22.6$; ESIMS: $\mathrm{m} / \mathrm{z} 150[\mathrm{M}+\mathrm{H}]^{+}, 172[\mathrm{M}+\mathrm{Na}]^{+}$; anal. Calcd for $\mathrm{C}_{9} \mathrm{H}_{11} \mathrm{NO}: \mathrm{C}$ 72.48; H 7.38; N 7.40; found: C 72.57; H 7.43; N 7.34.

$N$-(4-Hydroxyphenethyl) propionamide (Table 1, Entry 23):

IR $(\mathrm{KBr}): v=3417,1646,1551,1456,1275 \mathrm{~cm}^{-1} ;{ }^{1} \mathrm{H}$ NMR $\left(200 \mathrm{MHz}, \mathrm{CDCl}_{3}\right): \delta=9.54(1 \mathrm{H}, \mathrm{brs}), 7.01(2 \mathrm{H}, \mathrm{d}$, $J=8.0 \mathrm{~Hz}), 6.71(2 \mathrm{H}, \mathrm{d}, J=8.0 \mathrm{~Hz}), 5.42(1 \mathrm{H}, \mathrm{brs}), 3.49$ $(2 \mathrm{H}, \mathrm{q}, J=7.0 \mathrm{~Hz}) 2.80(2 \mathrm{H}, \mathrm{t}, J=7.0 \mathrm{~Hz}), 2.11(2 \mathrm{H}, \mathrm{q}, J$ $=7.0 \mathrm{~Hz}), 1.10(3 \mathrm{H}, \mathrm{t}, J=7.0 \mathrm{~Hz}) ;{ }^{13} \mathrm{C} \mathrm{NMR}(50 \mathrm{MHz}$, $\left.\mathrm{CDCl}_{3}\right): \delta=170.2,155.8,133.1,129.5,115.7,40.8,35.6$, 22.9, 20.9; ESIMS: m/z $194[\mathrm{M}+\mathrm{H}]^{+}, 216[\mathrm{M}+\mathrm{Na}]^{+}$; anal. Calcd for $\mathrm{C}_{11} \mathrm{H}_{15} \mathrm{NO}_{2}$ : C 68.39; $\mathrm{H} 7.77 ; \mathrm{N} \mathrm{7.25}$; found: $\mathrm{C}$ 68.28; H 7.83; N 7.17.

$N$-Phenethylacetamide (Table 1, Entry 24):

IR $(\mathrm{KBr}): v=3284,1652,1554,1451,1369,1296 \mathrm{~cm}^{-1}$;

${ }^{1} \mathrm{H}$ NMR $\left(200 \mathrm{MHz}, \mathrm{CDCl}_{3}\right): \delta=7.30-7.09(5 \mathrm{H}, \mathrm{m})$, $6.31(1 \mathrm{H}, \mathrm{brs}), 3.41(2 \mathrm{H}, \mathrm{q}, J=7.0 \mathrm{~Hz}), 2.79(2 \mathrm{H}, \mathrm{t}, J=$ $7.0 \mathrm{~Hz}), 1.90(3 \mathrm{H}, \mathrm{s}) ;{ }^{13} \mathrm{C} \mathrm{NMR}\left(50 \mathrm{MHz}, \mathrm{CDCl}_{3}\right): \delta=$ $170.2,138.9,128.7,128.5,126.4,40.8,35.6,22.9$; ESIMS: m/z $164[\mathrm{M}+\mathrm{H}]^{+}, 186[\mathrm{M}+\mathrm{Na}]^{+}$; anal. Calcd for $\mathrm{C}_{10} \mathrm{H}_{13} \mathrm{NO}$ : C 73.62; $\mathrm{H}$ 7.98; $\mathrm{N} \mathrm{8.59}$; found: $\mathrm{C} 73.73 ; \mathrm{H}$ 7.92; N 8.65.

$N$-Benzylpropionamide (Table 1, Entry 25):

IR $(\mathrm{KBr}): v=3293,1613,1553,1459,1381,1239 \mathrm{~cm}^{-1}$; ${ }^{1} \mathrm{H}$ NMR $\left(200 \mathrm{MHz}, \mathrm{CDCl}_{3}\right): \delta=7.37-7.11(5 \mathrm{H}, \mathrm{m})$, $5.65(1 \mathrm{H}$, brs $), 4.33(2 \mathrm{H}, \mathrm{s}), 2.11(2 \mathrm{H}, \mathrm{t}, J=7.0 \mathrm{~Hz}), 1.62$ $-1.53(2 \mathrm{H}, \mathrm{m}), 1.29-1.14(12 \mathrm{H}, \mathrm{m}), 0.83(3 \mathrm{H}, \mathrm{t}, J=7.0$ $\mathrm{Hz}) ;{ }^{13} \mathrm{C} \mathrm{NMR}\left(50 \mathrm{MHz}, \mathrm{CDCl}_{3}\right): \delta=171.5,127.8,127.5$, $128.7,128.6,127.4,127.3,65.1,44.6,43.6,36.8,31.8$, 29.5, 29.3, 25.8, 22.7, 14.1; ESIMS: m/z $262[\mathrm{M}+\mathrm{H}]^{+}$; anal. Calcd for $\mathrm{C}_{17} \mathrm{H}_{27} \mathrm{NO}$ : $\mathrm{C}$ 78.16; $\mathrm{H}$ 10.35; N 5.36; found: C 78.25; H 10.41; N 5.42.

\section{Acknowledgements}

The authors RAK and NS thank the CSIR for fellowship \& The author JK thank the UGC-DSK (New Delhi) for the Dr. D. S. Kothari fellowship (F. 4-2/2006 (BSR)/13-14/0087) and CSIR for financial assistance.

\section{References}

[1] I. L. Finar, Organic Chemistry, Vol. 1; The Fundamental Principles, $6^{\text {th }}$ Edn., ELBS and Longman Group Ltd., London, 1973, 658-665.

[2] S. R. Sandler, W. Karo. Organic Functional Group Preparations, $2^{\text {nd }}$ Edn., Academic Press, London, 1983, 1: 316-554.

[3] G. Benz. in. Comprehensive Organic Synthesis, Eds, Trost, B. M.; Fleming, I. Pergamon press, Oxford, 1991, 6, 381-417.

[4] C. Y. Duh, Y. C. Wu, S. K. Wang. Cytotoxic pyridone alkaloids from the leaves of Piper aborescens. J. Nat. Prod. 1990, 53 (6): 1575-1577.

[5] H-M. D. Wang, C-Y. Chen, P-F. Wu. Isophilippinolide A Arrests Cell Cycle Progression and Induces Apoptosis for Anticancer Inhibitory Agents in Human Melanoma Cells. J. Agric. Food Chem. 2014, 62 (5): 1057-1065.

[6] A. L. J. Beckwith. Synthesis of Amides in: The Chemistryof Amides, Ed. Zadrocki, J. Intersience, New York, 1970, 96-105.

[7] O-P. Andrea, G-S. Diego. Recent Developments in Amide Synthesis Using Nonactivated Starting Materials. J. Org. Chem. 2016, 81 (23): 11548-11555.

[8] K. Hyodo, G. Hasegawa, N. Oishi, K. Kuroda, K. Uchida. Direct and Catalytic Amide Synthesis from Ketones via Transoximation and Beckmann Rearrangement under Mild Conditions, J. Org. Chem. 2018, 83 (21): 13080-13087.

[9] C. Chen, Y. Zhang, S. H. Hong. N-Heterocyclic Carbene Based Ruthenium-Catalyzed Direct AmideSynthesis from Alcohols and Secondary Amines: Involvement of Esters, J. Org. Chem. 2011, 76 (24): 10005-10010.

[10] J. Cui, D. I. Chai, C. Miller, J. Hao, C. Thomas, J. Wang, K. A. Scheidt, S. A. Kozmin. Assembly of Four Diverse Heterocyclic Libraries Enabled by Prins Cyclization, AuCatalyzedEnyneCycloisomerization, and AutomatedAmide Synthesis. J. Org. Chem. 2012, 77 (17): 7435-7470.

[11] S. Masala, M. Taddei. Solid-Supported Chloro [1, 3, 5] triazine. A Versatile New SyntheticAuxiliary for the Synthesis of Amide Libraries. Org. Lett. 1999, 1 (9), 1355-1357

[12] Z. Fu, J. Lee, B. Kang, S. H. Hong . Dehydroge-native Amide Synthesis: Azide as a Nitrogen Source, Org. Lett. 2012, 14 (23): 6028-6031.

[13] J. Zhu, Y. Zhang, F. Shi, Y. Deng. Dehydrogenative amide synthesis from alcohol and amine catalyzed by hydrotalcitesupported gold nanoparticles. Tetrahedron Letters. 2012, 53 (25): 3178-3180.

[14] L. Zhang, X. J. Wang, J. Wang, N. Grinberg, D. K. Krishnamurthy, C. H. Senanayake. An improved method of amide synthesis using acyl chlorides. Tetrahedron Letters. 2009, 50 (24): 2964-2966. 
[15] L. E. Barstow, V. J. Hruby. Simple method for the synthesis of amides. J. Org. Chem. 1971, 36: 1305-1306.

[16] A. Garecka, M. leplawy, J. Zabrocki, A. Zwierzak. Diethyl Phosphorobromidate- An Effective New Peptide-Forming Agent. Synthesis. 1978, 6: 474-476.

[17] J. D. Meseguer, A. L. P. Coll, J. R. F. Lizabre, A. Z. Bilbao. A New Reagent for Activating Carboxyl Groups; Preparation and Reactions of $N, \quad N$-Bis [2-oxo-3-ox-azolidinyl] phosphorodiamidic Chloride. Synthesis. 1980, 7: 547-551.

[18] T. Kubota, S. Miyashita, T. Kitazume, N. Ishikawa. Novel synthetic reactions using bis (2, 2, 2-trifluoroethoxy) triphenylphosphorane. J. Org. Chem. 1980, 45 (25): 50525057.

[19] T. Kunieda, Y. Abe, T. Higuchi, M. Hirobe. A new reagent for activating carboxyl groups: diphenyl 2-oxo-3oxazolinylphosphonate. Tetrahedron Lett. 1981, 22: 12571258.

[20] G. Trapani, A. Reho, A. Latrofa. Trimethylamine-Borane as Useful Reagent in the $\mathrm{N}$-Acylation or $\mathrm{N}$-Alkylation of Amines by Carboxylic Acids. Synthesis. 1983, 12: 1013-1014.

[21] T. Ogawa, T. Hikasa, T. Ikegami, N. Ono, H. Suzuki. Selective activation of primary carboxylic acids by electron-rich triarylbismuthanes. Application to amide and ester synthesis under neutral conditions. J. Chem. Soc. Perkin Trans1. 1994, 3473-3478.

[22] I. Azumaya, T. Okamoto, F. Imabeppu, H. Takayanagi. Simple and convenient synthesis of tertiary benzanilides using dichlorotriphenylphosphorane. Tetrahedron. 2003, 59: 23252331.

[23] D. M. Shendge, R. Froehlich, G. Haufe. Highly Efficient Stereoconservative Amidation and Deamidation of $\alpha$-Amino Acids. Org. Lett. 2004, 6 (21): 3675-3678.

[24] M. Hossaini-Sarvave, H. Sharghi. ZnO as a New Catalyst for $\mathrm{N}$-Formylation of Amines under Solvent-Free Conditions. J. Org. Chem. 2006, 71 (17): 6652-6654.

[25] J. Bures, M. Martin, F. Urpi, J. Vilarrasa. Catalytic Staudinger-Vilarrasa Reaction for the Direct Ligation of Carboxylic Acids and Azides. J. Org. Chem. 2009, 74 (5): 2203-2206.

[26] B. Das, B. Venkataiah, A. Kashinatham. Venkatasin, A new CoumarinoLignoid from jatropha Gassypifolia. Nat. Prod. Lett. 1999, 13 (4): 293-297.

[27] B. Das, B. Venkataiah, P. Madhusudhan. A Simple and Efficient Selective Esterification of Aliphatic Carboxylic Acids in the Presence of Aromatic Carboxylic Acids. Synlett. 2000, 59-60.
[28] C. Ramesh, N. Ravindranath, B. Das. J. Org. Chem. 2003, 68 (18): 7101-7103.

[29] C. Ramesh, J. Benerji, R. Pal, B. Das. Silica Supported Sodium Hydrogen Sulfate and Amberlyst-15: Two Efficient Heterogeneous Catalysts for Facile Synthesis of Bis- and Tris (1H-indol-3-yl) methanes from Indoles and Carbonyl Compounds. Adv. Synth. Catal. 2003, 345 (5): 557-559.

[30] B. Das, K. Venkataiah, G. Mahender, I. Mahender. A simple and efficient method for $\alpha$-bromination of carbonyl compounds using $\mathrm{N}$-bromosuccinimide in the presence of silica-supported sodium hydrogen sulfate as a heterogeneous catalyst. Tetrahedron Lett. 2005, 46: 3041-3044.

[31] B. Das, K. R. Reddy, P. Thirupathi. A simple, efficient and highly selective deprotection of $t$-butyldimethylsilyl (TBDMS) ethers using silica supported sodium hydrogen sulfate as a heterogeneous catalyst. Tetrahedron Lett. 2006, 47: 5855 .

[32] B. Das, N. Chowddhury, K. Damodar, K. R. Reddy. Efficient Conjugate Addition of $1 H$-Indoles to Electron-Deficient Olefines Catalyzed by Silica-Supported Sodium Hydrogen Sulfate $\left(\mathrm{NaHSO}_{4} \cdot \mathrm{SiO}_{2}\right)$. Helv. Chem. Acta. 2007, 90: 340345 .

[33] B. Das, Ch. R. Reddy, D. N. Kumar, M. Krishnaiah, R. Narender. A Simple, Advantageous Synthesis of 5-Substituted 1H-Tetrazoles. Synlett. 2010, 391-394.

[34] K. Boroczky, H. Laatsch, I. Wagner-Dobler, K. Stritzke, S. Schulz. Chem analysis as selection and dereplication tool for the identification of new natural compounds from large sample sets. Chem. Biodiversity. 2006, 3: 622-634.

[35] T. H. Jones, H. M. Garraffo, T. F. Spande, N. R. Andriamaharavo, J. S. T. Gorman, A. J. Snyder, A. W. Jeter, J. A. Torres, R. R. Snelling, J. W. Daly. Caste-Specific Tyramides from Myrmicine Ants. J. Nat. Prod. 2010, 71 (3): 313-316.

[36] Z. J. Lin, X. M. Lu, T. J. Zhu, Y. C. Fang, Q. Q. Gu, W. Zhu. GPR12 selections of the metabolites from an endophytic Streptomyces sp. associated with Cistanches deserticola. Arch. Pharm. Res. 2008, 31 (9): 1108-1114.

[37] N. Ullah, K. M. Arafch. The first total synthesis of aplysamine 6 , an inhibitor of isoprenylcysteine carboxy methyltransferase. Tetrahedron Lett. 2009, 50: 158-160.

[38] A. S. B. Prasad, J. V. B. Kanth, M. Periasamy. Convenient methods for the reduction of amides, nitriles, carboxylic esters, acids and hydroboration of alkenes using $\mathrm{NaBH}_{4} / \mathrm{I}_{2}$ system. Tetrahedron. 1992, 48: 4623-4628.

[39] G. W. Breton. Selective Monoacetylation of Unsymmetrical Diols Catalyzed by Silica Gel-Supported Sodium Hydrogen Sulfate. J. Org. Chem. 1997, 62 (25): 8952-8954. 